HETEROCYCLES, Vol. , No. , , pp. -. (c) The Japan Institute of Heterocyclic Chemistry

Received, , Accepted, , Published online, . COM-06- (Please do not delete.)

\title{
PREPARATION OF 5H,7H-DIBENZ[c,e]OXEPIN-5-ONE DERIVATIVE THROUGH RECONSTRUCTION OF THE LACTONE RING
}

\author{
Hitoshi Abe, ${ }^{a}$ Masatsugu Arai, ${ }^{b}$ Yasuo Takeuchi, ${ }^{b}$ and \\ Takashi Harayama ${ }^{\mathrm{c} *}$
}

a) Graduate School of Science and Engineering, University of Toyama, Gofuku 3190, Toyama 930-8555, Japan, b) Faculty of Pharmaceutical Sciences, Okayama University, Okayama 700-8530, Japan, c) Faculty of Pharmaceutical Sciences at Kagawa Campus, Tokushima Bunri University, Sanuki, Kagawa 796-2193, Japan abeh@eng.u-toyama.ac.jp; harayama@kph.bunri-u.ac.jp

\begin{abstract}
The systematic investigation of the efficient transformation of $6 H$-dibenzo[b,d]pyran-6-one into $5 H, 7 H$-dibenz[c,e $]$ oxepin-5-one is described.
\end{abstract}

The $5 H, 7 H$-dibenz [c,e]oxepin-5-one skeleton is a structurally rare ring system among natural products. ${ }^{1}$ However, recent research has shown that the naturally occurring compounds containing this ring system exhibit quite interesting biological activities, such as anti-tumor ${ }^{1 \mathrm{~d}}$ and tyrosine kinase inhibiting properties. $^{1 \mathrm{~b}, 1 \mathrm{c}}$

Based on such informaion, we have demonstrated the short-step syntheses of the compounds, graphislactone $\mathrm{D}^{2}$ and ulocladol, ${ }^{3}$ using a palladium-mediated biaryl coupling reaction. ${ }^{4}$ In our synthetic route for these compounds, a lactone reconstruction process was the key step for the formation of the $5 H$,7H-dibenz[c,e $]$ oxepin-5-one skeleton (Scheme 1). ${ }^{5}$ Although this useful transformation is thought to be applicable for other synthetic uses, there has been no systematic investigation of this type of transformation. In this report, we describe the scope and limitations of the ring transformation of the 6H-dibenzo[b,d]pyran-6-one into 5H,7H-dibenz[c,e]oxepin-5-one.

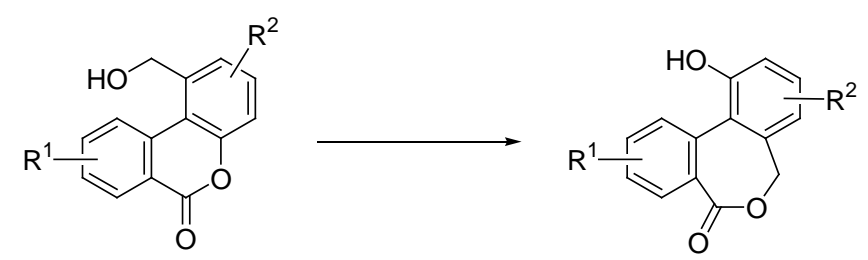


We initially examined the reaction of the six-membered ring lactone $\mathbf{1}^{6}$ as a substrate using various alcohols (Table 1). Under elevated temperature conditions, 1 was transformed into a seven-membered ring lactone $\mathbf{2}$ accompanied by the ring opened products $\mathbf{3}$ and $\mathbf{4}$ when normal-alkyl alcohols were used (Runs 1-3). On the other hand, when the branched alcohols were employed, the lactone-opened biphenyls 3 and $\mathbf{4}$ were mainly generated (Run 4). Especially, when secondary alcohol was employed, the acetoxy compound 4 was exclusively formed (Runs 5 and 6).

Table1. Transformation of lactone compound 1

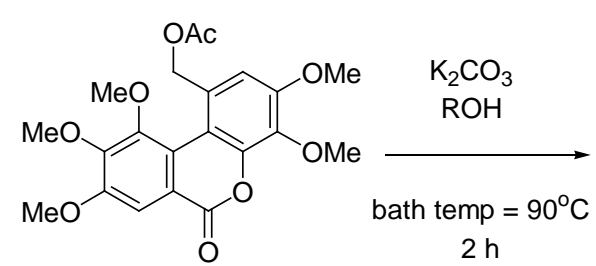

1

\begin{tabular}{ccccc}
\hline \multirow{2}{*}{ Run } & $\mathrm{R}$ & \multicolumn{3}{c}{ Yield (\%) } \\
\cline { 3 - 5 } & & $\mathbf{2}$ & $\mathbf{3}$ & $\mathbf{4}$ \\
\hline 1 & $\mathrm{Me}$ & 59 & 39 & - \\
2 & $\mathrm{Et}$ & 44 & 38 & - \\
3 & ${ }^{n} \mathrm{Bu}$ & 35 & 42 & 16 \\
4 & ${ }^{i} \mathrm{Bu}$ & 8 & 18 & 54 \\
5 & ${ }^{i} \mathrm{Pr}$ & - & - & 93 \\
6 & ${ }^{{ }^{n} c} \mathrm{Bu}$ & - & - & 73 \\
\hline
\end{tabular}<smiles>COc1cc2c(c(OC)c1OC)-c1c(cc(OC)c(OC)c1OC)C(=O)OC2</smiles>

2<smiles>[R]OCc1cc(OC)c(OC)c(OC)c1-c1c(CO)cc(OC)c(OC)c1O</smiles>

3a: $R=M e$

3b: $R=E t$

3c: $\mathrm{R}={ }^{n} \mathrm{Bu}$ 3d: $\mathrm{R}={ }^{i} \mathrm{Bu}$<smiles>[R]OC(=O)c1cc(OC)c(OC)c(OC)c1-c1c(OC)cc(OC)c(OC)c1OC</smiles>

4c: $\mathrm{R}={ }^{n} \mathrm{Bu}$

4d: $\mathrm{R}={ }^{i} \mathrm{Bu}$

4e: $\mathrm{R}={ }^{i} \mathrm{Pr}$

4f: $R={ }^{s e c} B u$

These results suggest that the reactivity of this transformation is related to the size of the alkyl moiety of the alcohol. The acetoxy group can be easily removed by methanol or ethanol, followed by the lactone formation to afford the seven-membered ring (Runs 1 and 2). However, it seems different from the previously reported ones, namely, as illustrated in Scheme 2, similar transformations successfully produced the corresponding seven-membered ring lactone compounds in high yields without generating the biphenyls. ${ }^{2,3}$<smiles>COc1cc(O)c2c(=O)oc3c(OCc4ccccc4)c(OC)cc(CO)c3c2c1</smiles>

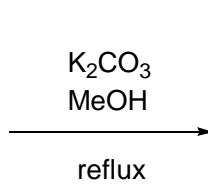<smiles>[2H]c1cc(OC)cc2c1C(=O)OCc1cc(OC)c(OC)c(O)c1-2</smiles>

$\mathrm{R}=\mathrm{Me}(85 \%)$

$\mathrm{R}=\mathrm{Bn}(82 \%)$

Scheme 2. Previously reported transformation of the lactones

Based on the difference in the above reactivity, the presence of a substituent on the 10-position might be 
crucial for this conversion. In order to clarify the importance of the 10-position, we examined the same reaction using the 10-demethoxy compound 5, which was prepared by the simple esterification between carboxylic acid $\mathbf{6}^{7}$ and phenol $7^{8}$ followed by the intramolecular coupling reaction (Scheme 3). During the reaction of $\mathbf{5}$ with the methanol and potassium carbonate system, the expected transformation into the seven-membered ring lactone $\mathbf{8}$ took place without the generation of the corresponding biphenyl (Scheme 4).

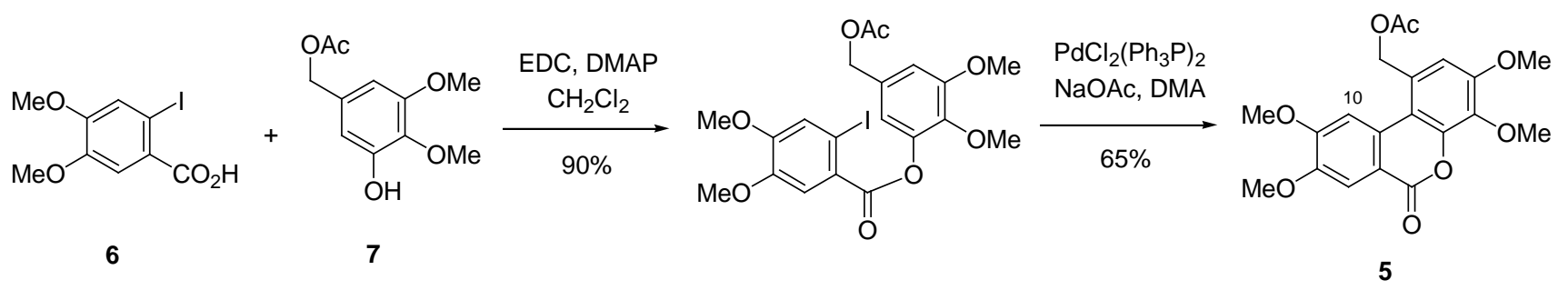

Scheme 3. Preparation of six-membered ring lactone $\mathbf{5}$

In summary, we investigated the facile ring transformation of $6 \mathrm{H}$-dibenzo[b,d]pyran-6-one into $5 H, 7 H$-dibenz $[c, e]$ oxepin-5-one in the presence of an alcohol under basic conditions. In this reaction, the absence of the substituent at the 10-position of the $6 \mathrm{H}$-dibenzo[b,d]pyran-6-one system was essential for the formation of the desired seven-membered ring lactone.
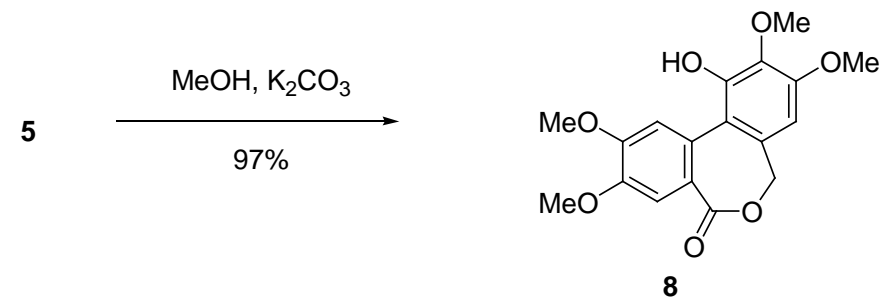

Scheme 4. Transformation of $\mathbf{5}$ into seven-membered ring lactone $\mathbf{8}$

\section{EXPERIMENTAL}

General: Melting points were measured using a Yanagimoto micro melting point hot-plate apparatus and are uncorrected. The IR spectra were recorded using a JASCO FTIR-350 spectrophotometer. The NMR spectra were obtained using a Varian MERCURY-300 or VXR-500 instrument with the chemical shifts being reported in $\delta$ ppm. The FAB-MS was obtained using a VG-Autospec instrument with $m$-nitrobenzyl alcohol as the matrix. Silica-gel column chromatography was carried out using Merck 9385 Kieselgel 60.

\section{General procedure for the ring transformation (Table 1)}

A mixture of 1 (280 mg, $0.674 \mathrm{mmol}$ ), potassium carbonate (187 mg, $1.35 \mathrm{mmol}$ ), and an alcohol (15 $\mathrm{mL}$ ) was heated at $90{ }^{\circ} \mathrm{C}$ with stirring for $2 \mathrm{~h}$. The reaction mixture was poured into water and 
extracted with EtOAc. The organic layer was washed with brine, dried over magnesium sulfate, and evaporated to give a residue, which was purified by silica-gel column chromatography using hexane-EtOAc as the eluent.

\section{1-Hydroxy-1,2,3,9,10-pentamethoxy-5H,7H-dibenz[c,e]oxepin-5-one (2)}

Colorless needles, mp $205^{\circ} \mathrm{C}\left(\mathrm{CH}_{2} \mathrm{Cl}_{2}\right.$-hexane). ${ }^{1} \mathrm{H}-\mathrm{NMR}\left(300 \mathrm{MHz}, \mathrm{CDCl}_{3}\right) \delta: 3.77\left(3 \mathrm{H}, \mathrm{s}, \mathrm{OCH}_{3}\right)$, $3.928\left(3 \mathrm{H}, \mathrm{s}, \mathrm{OCH}_{3}\right), 3.930\left(3 \mathrm{H}, \mathrm{s}, \mathrm{OCH}_{3}\right), 3.95\left(3 \mathrm{H}, \mathrm{s}, \mathrm{OCH}_{3}\right), 3.98\left(3 \mathrm{H}, \mathrm{s}, \mathrm{OCH}_{3}\right), 4.81(1 \mathrm{H}, \mathrm{d}, \mathrm{A}$ of $\mathrm{AB}$, $\left.J=11.7 \mathrm{~Hz}, \mathrm{ArCH}_{\mathrm{A}} \mathrm{H}_{\mathrm{B}} \mathrm{O}\right), 4.97\left(1 \mathrm{H}, \mathrm{d}, \mathrm{B}\right.$ of $\left.\mathrm{AB}, J=11.7 \mathrm{~Hz}, \operatorname{ArCH}_{\mathrm{A}} H_{B} \mathrm{O}\right), 6.64(1 \mathrm{H}, \mathrm{s}, 8-\mathrm{H}), 6.70(1 \mathrm{H}, \mathrm{s}$, $\mathrm{OH}$, exchangeable with $\left.\mathrm{D}_{2} \mathrm{O}\right), 7.22(1 \mathrm{H}, \mathrm{s}, 4-\mathrm{H}) .{ }^{13} \mathrm{C}-\mathrm{NMR}\left(75 \mathrm{MHz}, \mathrm{CDCl}_{3}\right) \delta: 56.1,56.3,61.1,61.2$, 61.9, 69.9, 104.7, 109.9, 115.8, 120.9, 127.1, 131.2, 138.0, 145.1, 148.3, 149.9, 152.4, 153.0, 169.6.

IR (KBr) cm $\mathrm{cm}^{-1}: 3400(\mathrm{OH}), 1720(\mathrm{C}=\mathrm{O})$, 1460, 1400, 1350, 1330, 1150, 1110 (O-C=O), 1000. FAB-Mass (positive ion mode) $\mathrm{m} / \mathrm{z}: 376[\mathrm{M}]^{+}, 377[\mathrm{M}+1]^{+}$. HRMS (FAB) calcd for $\mathrm{C}_{19} \mathrm{H}_{20} \mathrm{O}_{8}[\mathrm{M}]^{+}$: 376.1158. Found: 376.1131. Anal. Calcd for $\mathrm{C}_{19} \mathrm{H}_{20} \mathrm{O}_{8}$ : C, 60.63; H, 5.36. Found: C, 60.35; H, 5.32.

2-Hydroxy-6-hydroxymethyl-2',3,3',4,4'-pentamethoxy-6'-methoxycarbonyl-1,1'-biphenyl (3a) Colorless needles, mp 143-144 ${ }^{\circ} \mathrm{C}\left(\mathrm{CH}_{2} \mathrm{Cl}_{2}\right.$-hexane). ${ }^{1} \mathrm{H}-\mathrm{NMR}\left(300 \mathrm{MHz}, \mathrm{CDCl}_{3}\right) \delta$ : $3.57(3 \mathrm{H}, \mathrm{s}$, $\left.\mathrm{COOCH}_{3}\right), 3.66\left(3 \mathrm{H}, \mathrm{s}, \mathrm{OCH}_{3}\right), 3.91\left(3 \mathrm{H}, \mathrm{s}, \mathrm{OCH}_{3}\right), 3.94\left(3 \mathrm{H}, \mathrm{s}, \mathrm{OCH}_{3}\right), 3.95\left(3 \mathrm{H}, \mathrm{s}, \mathrm{OCH}_{3}\right), 3.96(3 \mathrm{H}, \mathrm{s}$, $\left.\mathrm{OCH}_{3}\right), 4.22\left(1 \mathrm{H}, \mathrm{d}, \mathrm{A}\right.$ of $\left.\mathrm{AB}, J=11.6 \mathrm{~Hz}, \mathrm{ArCH}_{A} \mathrm{H}_{\mathrm{B}} \mathrm{OH}\right), 4.27(1 \mathrm{H}, \mathrm{d}, \mathrm{B}$ of $\mathrm{AB}, J=11.6 \mathrm{~Hz}$, $\left.\mathrm{ArCH}_{\mathrm{A}} \mathrm{H}_{\mathrm{B}} \mathrm{OH}\right), 5.72$ (1H, bs, Ar-OH, exchangeable with $\left.\mathrm{D}_{2} \mathrm{O}\right), 6.71(1 \mathrm{H}, \mathrm{s}, 5-\mathrm{H}), 7.34(1 \mathrm{H}, \mathrm{s}, 5$ ' $-\mathrm{H})$. ${ }^{13} \mathrm{C}-\mathrm{NMR}\left(75 \mathrm{MHz}, \mathrm{CDCl}_{3}\right.$ ) $\delta: 52.3,55.7,56.1,61.05,61.08,61.13,64.2,104.6,109.6,115.2,124.1$, 126.5, 134.5, 135.1, 145.7, 146.3, 151.4, 151.5, 152.4, 167.3. IR (KBr) cm $\mathrm{cm}^{-1}: 3500(\mathrm{OH}), 3340(\mathrm{OH})$, $1720(\mathrm{C}=\mathrm{O}), 1100(\mathrm{O}-\mathrm{C}=\mathrm{O})$. FAB-Mass (positive ion mode) m/z: $408[\mathrm{M}]^{+}$. Anal. Calcd for $\mathrm{C}_{20} \mathrm{H}_{24} \mathrm{O}_{9}$ : C, 58.82; H, 5.92. Found: C, 58.64; H, 5.92.

\section{6-Ethoxycarbonyl-2'-hydroxy-6'-hydroxyethyl-2,3,3',4,4'-pentamethoxy-1,1'-biphenyl (3b)}

Colorless needles, mp 146-147 ${ }^{\circ} \mathrm{C}\left(\mathrm{CH}_{2} \mathrm{Cl}_{2}\right.$-hexane). ${ }^{1} \mathrm{H}-\mathrm{NMR}\left(300 \mathrm{MHz}, \mathrm{CDCl}_{3}\right) \delta: 1.05(3 \mathrm{H}, \mathrm{t}, J=7.2$ $\left.\mathrm{Hz}, \mathrm{COOCH}_{2} \mathrm{CH}_{3}\right), 3.57\left(3 \mathrm{H}, \mathrm{s}, \mathrm{OCH}_{3}\right), 3.91\left(3 \mathrm{H}, \mathrm{s}, \mathrm{OCH}_{3}\right), 3.93\left(3 \mathrm{H}, \mathrm{s}, \mathrm{OCH}_{3}\right), 3.95\left(3 \mathrm{H}, \mathrm{s}, \mathrm{OCH}_{3}\right)$, $3.96\left(3 \mathrm{H}, \mathrm{s}, \mathrm{OCH}_{3}\right), 4.08\left(2 \mathrm{H}, \mathrm{q}, J=7.2 \mathrm{~Hz}, \mathrm{COOCH}_{2} \mathrm{CH}_{3}\right), 4.24(1 \mathrm{H}, \mathrm{d}, \mathrm{A}$ of $\mathrm{AB}, J=11.4 \mathrm{~Hz}$, $\left.\mathrm{ArCH}_{A} \mathrm{H}_{\mathrm{B}} \mathrm{OH}\right), 4.25\left(1 \mathrm{H}, \mathrm{d}, \mathrm{B}\right.$ of $\left.\mathrm{AB}, J=11.4 \mathrm{~Hz}, \mathrm{ArCH}_{\mathrm{A}} \mathrm{H}_{B} \mathrm{OH}\right), 5.75(1 \mathrm{H}$, bs, Ar-OH, exchangeable with $\left.\mathrm{D}_{2} \mathrm{O}\right), 6.70\left(1 \mathrm{H}, \mathrm{s}, 5\right.$ '-H), $7.33(1 \mathrm{H}, \mathrm{s}, 5-\mathrm{H}) .{ }^{13} \mathrm{C}-\mathrm{NMR}\left(75 \mathrm{MHz}, \mathrm{CDCl}_{3}\right) \delta: 13.9,55.7,56.1,61.0$, 61.1, 61.1, 64.1, 104.5, 109.5, 115.4, 123.6, 127.2, 134.5, 135.1, 145.5, 146.4, 151.3, 151.4, 152.5, 167.1. IR (KBr) cm cm $^{-1} 3360(\mathrm{OH}), 3200(\mathrm{OH}), 1690(\mathrm{C}=\mathrm{O}), 1110(\mathrm{O}-\mathrm{C}=\mathrm{O})$. Anal. Calcd for $\mathrm{C}_{21} \mathrm{H}_{26} \mathrm{O}_{9}$ : C, 59.71; H, 6.20. Found: C, 59.63; H, 6.02.

\section{6-Butoxycarbonyl-2'-hydroxy-6'-hydroxymethyl-2,3,3',4,4'-pentamethoxy-1,1'-biphenyl (3c)}

Colorless needles, mp 126-127 ${ }^{\circ} \mathrm{C}\left(\mathrm{CH}_{2} \mathrm{Cl}_{2}\right.$-hexane). ${ }^{1} \mathrm{H}-\mathrm{NMR}\left(300 \mathrm{MHz}, \mathrm{CDCl}_{3}\right) \delta: 0.85(3 \mathrm{H}, \mathrm{t}, J=7.2$ $\left.\mathrm{Hz}, \mathrm{CH}_{2} \mathrm{CH}_{3}\right), 1.20$ (2H, sxt, $\left.J=7.5 \mathrm{~Hz}, \mathrm{CH}_{2} \mathrm{CH}_{2} \mathrm{CH}_{3}\right), 1.33-1.45\left(2 \mathrm{H}, \mathrm{m}, \mathrm{CH}_{2} \mathrm{CH}_{2} \mathrm{CH}_{2}\right), 2.81(1 \mathrm{H}, \mathrm{bs}$, $\left.\mathrm{CH}_{2} \mathrm{OH}\right), 3.56\left(3 \mathrm{H}, \mathrm{s}, 2-\mathrm{OCH}_{3}\right), 3.91\left(3 \mathrm{H}, \mathrm{s}, \mathrm{OCH}_{3}\right), 3.93\left(3 \mathrm{H}, \mathrm{s}, \mathrm{OCH}_{3}\right), 3.95\left(3 \mathrm{H}, \mathrm{s}, \mathrm{OCH}_{3}\right), 3.96(6 \mathrm{H}, \mathrm{s}$, 
$\left.2 \times \mathrm{OCH}_{3}\right), 4.03\left(2 \mathrm{H}, \mathrm{td}, J=3.0,6.6 \mathrm{~Hz}, \mathrm{COOCH}_{2}\right), 4.24\left(2 \mathrm{H}, \mathrm{s}, \mathrm{CH}_{2} \mathrm{OH}\right), 5.73(1 \mathrm{H}, \mathrm{s}, \mathrm{Ar}-\mathrm{OH}), 6.70(1 \mathrm{H}$, s, 5'-H), 7.34 (1H, s, 5-H). ${ }^{13} \mathrm{C}-\mathrm{NMR}\left(75 \mathrm{MHz} \mathrm{CDCl}_{3}\right) \delta: 13.8,19.1,30.5,55.7,56.1,61.0,61.09$, 61.14, 64.2, 65.1, 104.6, 109.6, 115.4, 123.6, 127.2, 134.5, 135.2, 145.5, 146.4, 151.3, 151.4, 152.5, 167.3. IR (KBr) cm ${ }^{-1}: 3400(\mathrm{OH}), 3300(\mathrm{OH}), 1700(\mathrm{C}=\mathrm{O}), 1460,1340,1230,1125,1110,1000$. Anal. Calcd for $\mathrm{C}_{23} \mathrm{H}_{30} \mathrm{O}_{9}$ : C, 61.32; H, 6.71. Found: C, 61.26; H, 6.57.

6-Acetoxymethyl-6'-butoxycabonyl-2-hydroxy-2',3,3',4,4'-pentamethoxy-1,1'-biphenyl (4c)

Pale yellow oil. ${ }^{1} \mathrm{H}-\mathrm{NMR}\left(500 \mathrm{MHz}, \mathrm{CDCl}_{3}\right) \delta: 0.82\left(3 \mathrm{H}, \mathrm{t}, J=7.3 \mathrm{~Hz}, \mathrm{CH}_{2} \mathrm{CH}_{3}\right), 1.13(2 \mathrm{H}, \mathrm{sxt}, J=7.5$ $\mathrm{Hz}, \mathrm{CH}_{2} \mathrm{CH}_{2} \mathrm{CH}_{3}$ ), 1.32 (2H, quint, $\left.J=7.0 \mathrm{~Hz}, \mathrm{CH}_{2} \mathrm{CH}_{2} \mathrm{CH}_{2}\right), 1.97\left(3 \mathrm{H}, \mathrm{s}, \mathrm{OCOCH}_{3}\right), 3.62(3 \mathrm{H}, \mathrm{s}$, 2'- $\left.\mathrm{OCH}_{3}\right), 3.92\left(3 \mathrm{H}, \mathrm{s}, \mathrm{OCH}_{3}\right), 3.93\left(3 \mathrm{H}, \mathrm{s}, \mathrm{OCH}_{3}\right), 3.94\left(6 \mathrm{H}, \mathrm{s}, 2 \times \mathrm{OCH}_{3}\right), 3.99(2 \mathrm{H}, \mathrm{td}, J=2.7,5.4 \mathrm{~Hz}$, $\left.\mathrm{COOCH}_{2}\right), 4.65\left(1 \mathrm{H}, \mathrm{d}, \mathrm{A}\right.$ of $\left.\mathrm{AB}, J=12.5 \mathrm{~Hz}, \mathrm{CH}_{A} \mathrm{H}_{\mathrm{B}} \mathrm{OAc}\right), 4.78(1 \mathrm{H}, \mathrm{d}, \mathrm{B}$ of $\mathrm{AB}, J=12.5 \mathrm{~Hz}$, $\mathrm{CH}_{\mathrm{A}} \mathrm{H}_{\mathrm{B}} \mathrm{OAc}$ ), 5.77 (1H, s, Ar-OH), $6.60(1 \mathrm{H}, \mathrm{s}, 5-\mathrm{H}), 7.39$ (1H, s, 5'-H). ${ }^{13} \mathrm{C}-\mathrm{NMR}\left(75 \mathrm{MHz}, \mathrm{CDCl}_{3}\right) \delta$ : 13.8, 19.1, 21.0, 30.5, 55.8, 56.1, 60.87, 60.91, 61.0, 64.79, 64.84, 104.5, 109.7, 117.3, 123.1, 126.7, 129.7, 134.9, 145.5, 146.7, 151.1, 151.7, 152.6, 166.7, 170.5. IR (neat) $\mathrm{cm}^{-1}: 3420(\mathrm{OH}), 1740(\mathrm{C}=\mathrm{O})$, 1700 (C=O), 1590, 1490, 1460, 1330, 1230, 1100, 1000. FAB-Mass (positive ion mode) m/z: $492[\mathrm{M}]^{+}$.

\section{6-(2-Methylpropoxy)carbonyl-2'-hydroxy-6'-hydroxymethyl-2,3,3',4,4'-pentamethoxy-1,1'-}

\section{biphenyl (3d)}

Colorless oil. ${ }^{1} \mathrm{H}-\mathrm{NMR}\left(300 \mathrm{MHz}, \mathrm{CDCl}_{3}\right) \delta: 0.79\left(3 \mathrm{H}, \mathrm{d}, J=4.8 \mathrm{~Hz},-\mathrm{CH}_{3}\right), 0.81(3 \mathrm{H}, \mathrm{d}, J=4.5 \mathrm{~Hz}$, $\left.-\mathrm{CH}_{3}\right), 1.73\left(2 \mathrm{H}\right.$, sep, $\left.J=6.6 \mathrm{~Hz}, \mathrm{CH}_{2} \mathrm{CH}\left(\mathrm{CH}_{3}\right)_{2}\right), 3.56\left(3 \mathrm{H}, \mathrm{s}, \mathrm{OCH}_{3}\right), 3.77-3.91(2 \mathrm{H}, \mathrm{m}, J=6.6 \mathrm{~Hz}$, $\left.\mathrm{COOCH}_{2} \mathrm{CH}\right), 3.90$ (3H, s, $\left.\mathrm{OCH}_{3}\right), 3.93\left(3 \mathrm{H}, \mathrm{s}, \mathrm{OCH}_{3}\right), 3.95\left(6 \mathrm{H}, \mathrm{s}, \mathrm{OCH}_{3}\right), 3.96\left(6 \mathrm{H}, \mathrm{s}, \mathrm{OCH}_{3}\right), 4.23(1 \mathrm{H}$, d, A of $\left.\mathrm{AB}, J=11.7 \mathrm{~Hz}, \mathrm{ArCH}_{\mathrm{A}} \mathrm{H}_{\mathrm{B}} \mathrm{OH}\right), 4.27\left(1 \mathrm{H}, \mathrm{d}, \mathrm{B}\right.$ of $\left.\mathrm{AB}, J=11.7 \mathrm{~Hz}, \operatorname{ArCH}_{\mathrm{A}} H_{B} \mathrm{OH}\right), 5.71(1 \mathrm{H}, \mathrm{s}$, $\mathrm{Ar}-\mathrm{OH}), 6.70\left(1 \mathrm{H}, \mathrm{s}, 5\right.$ '-H), $7.36(1 \mathrm{H}, \mathrm{s}, 5-\mathrm{H}) .{ }^{13} \mathrm{C}-\mathrm{NMR}\left(75 \mathrm{MHz}, \mathrm{CDCl}_{3}\right) \delta: 18.9,19.0,55.7,56.0$, 60.9, 61.0, 61.1, 64.1, 71.3, 104.7, 109.7, 115.4, 123.8, 127.2, 134.6, 135.3, 145.6, 146.5, 151.48, 151.54, 152.6, 167.4. IR $\left(\mathrm{CHCl}_{3}\right) \mathrm{cm}^{-1}: 3520(\mathrm{OH}), 1700(\mathrm{C}=\mathrm{O}), 1460,1340,1220,1100$. FAB-Mass (positive ion mode) $\mathrm{m} / \mathrm{z}: 492[\mathrm{M}]^{+}$.

\section{6-Acetoxymethyl-6'-(2-methylpropoxy)carbonyl-2-hydroxy-2',3,3',4,4'-pentamethoxy-1,1'-biphenyl} (4d)

Colorless oil. ${ }^{1} \mathrm{H}-\mathrm{NMR}\left(500 \mathrm{MHz}, \mathrm{CDCl}_{3}\right) \delta: 0.75\left(3 \mathrm{H}, \mathrm{s},-\mathrm{CH}_{3}\right), 0.77$ (3H, s, $\left.-\mathrm{CH}_{3}\right), 1.65$ (1H, sep, $J=$ $\left.6.6 \mathrm{~Hz}, \mathrm{CH}_{2} \mathrm{CH}\left(\mathrm{CH}_{3}\right)_{2}\right), 1.96\left(3 \mathrm{H}, \mathrm{s}, \mathrm{OCOCH}_{3}\right), 3.62\left(3 \mathrm{H}, \mathrm{s}, \mathrm{OCH}_{3}\right), 3.79(2 \mathrm{H}, \mathrm{d}, J=6.6 \mathrm{~Hz}$, $\left.\mathrm{COOCH}_{2} \mathrm{CH}\right), 3.92\left(3 \mathrm{H}, \mathrm{s}, \mathrm{OCH}_{3}\right), 3.93\left(3 \mathrm{H}, \mathrm{s}, \mathrm{OCH}_{3}\right), 3.95\left(6 \mathrm{H}, \mathrm{s}, \mathrm{ArOCH}_{3}\right), 4.66(1 \mathrm{H}, \mathrm{d}, \mathrm{A}$ of $\mathrm{AB}, J=$ $\left.12.0 \mathrm{~Hz}, \mathrm{ArCH}_{A} \mathrm{H}_{\mathrm{B}} \mathrm{OAc}\right), 4.79\left(1 \mathrm{H}, \mathrm{d}, \mathrm{B}\right.$ of $\left.\mathrm{AB}, J=12.0 \mathrm{~Hz}, \mathrm{ArCH}_{\mathrm{A}} \mathrm{H}_{B} \mathrm{OAc}\right), 5.77$ (1H, s, Ar-OH), 6.61

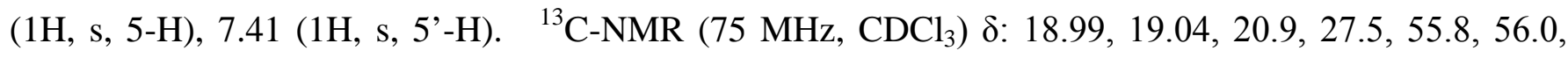
60.8, 60.85, 60.91, 64.8, 71.3, 104.7, 109.8, 117.3, 123.3, 126.7, 129.7, 135.1, 145.6, 146.8, 151.2, 151.9, 152.7, 166.8, 170.7. IR $\left(\mathrm{CHCl}_{3}\right) \mathrm{cm}^{-1}: 3520(\mathrm{OH}), 1730(\mathrm{C}=\mathrm{O}), 1700(\mathrm{C}=\mathrm{O}), 1460,1330,1240,1110$. FAB-Mass (positive ion mode) $\mathrm{m} / \mathrm{z}: 450[\mathrm{M}]^{+}$. 
6-Acetoxymethyl-2-hydroxy-6'-isopropoxycarbonyl-2',3,3',4,4'-pentamethoxy-1,1'-biphenyl (4e)

Colorless needles, mp 123-124 ${ }^{\circ} \mathrm{C}\left(\mathrm{CH}_{2} \mathrm{Cl}_{2}\right.$-hexane). ${ }^{1} \mathrm{H}-\mathrm{NMR}\left(300 \mathrm{MHz}, \mathrm{CDCl}_{3}\right) \delta: 0.92(3 \mathrm{H}, \mathrm{d}, J=6.0$ $\left.\mathrm{Hz}, \mathrm{CH}\left(\mathrm{CH}_{3}\right)_{2}\right), 1.01\left(3 \mathrm{H}, \mathrm{d}, J=6.0 \mathrm{~Hz}, \mathrm{CH}\left(\mathrm{CH}_{3}\right)_{2}\right), 1.98\left(3 \mathrm{H}, \mathrm{s}, \mathrm{OCOCH}_{3}\right), 3.65\left(3 \mathrm{H}, \mathrm{s}, \mathrm{OCH}_{3}\right), 3.92$ $\left(3 \mathrm{H}, \mathrm{s}, \mathrm{OCH}_{3}\right), 3.94\left(3 \mathrm{H}, \mathrm{s}, \mathrm{OCH}_{3}\right), 3.94\left(6 \mathrm{H}, \mathrm{s}, 2 \times \mathrm{OCH}_{3}\right), 4.66(1 \mathrm{H}, \mathrm{d}, \mathrm{A}$ of $\mathrm{AB}, J=12.3 \mathrm{~Hz}$, $\left.\mathrm{ArCH}_{A} \mathrm{H}_{\mathrm{B}} \mathrm{OAc}\right), 4.76\left(1 \mathrm{H}, \mathrm{d}, \mathrm{B}\right.$ of $\left.\mathrm{AB}, J=12.3 \mathrm{~Hz}, \operatorname{ArCH}_{\mathrm{A}} H_{B} \mathrm{OAc}\right), 4.92(1 \mathrm{H}$, sep, $J=6.3 \mathrm{~Hz}$, $\left.\mathrm{CH}\left(\mathrm{CH}_{3}\right)_{2}\right), 5.75(1 \mathrm{H}, \mathrm{s}, \mathrm{Ar}-\mathrm{OH}), 6.60(1 \mathrm{H}, \mathrm{s}, 5-\mathrm{H}), 7.36\left(1 \mathrm{H}, \mathrm{s}, 5\right.$ '-H). ${ }^{13} \mathrm{C}-\mathrm{NMR}\left(75 \mathrm{MHz}, \mathrm{CDCl}_{3}\right) \delta$ : 21.0, 21.4, 21.5, 55.9, 56.1, 60.9, 61.0, 64.8, 67.9, 104.4, 109.5, 117.5, 122.7, 127.4, 129.8, 134.9, 145.3, 146.8, 151.1, 151.7, 152.7, 166.3, 170.6. IR (KBr) cm cm $^{-1} 3520(\mathrm{OH}), 1730(\mathrm{C}=\mathrm{O}), 1695(\mathrm{C}=\mathrm{O}), 1360$, 1250, 1105. Anal. Calcd for $\mathrm{C}_{24} \mathrm{H}_{30} \mathrm{O}_{10}$ : C, 60.24; H, 6.32. Found: C, 60.25; H, 6.25.

\section{6-Acetoxymethyl-6'-sec-butoxycarbonyl-2-hydroxy-2',3,3',4,4'-pentamethoxy-1,1'-biphenyl (4f)}

1:1 Mixture of diastereomers. Colorless needles, mp 91-94 ${ }^{\circ} \mathrm{C}\left(\mathrm{CH}_{2} \mathrm{Cl}_{2}\right.$-hexane). ${ }^{1} \mathrm{H}-\mathrm{NMR}(300 \mathrm{MHz}$, $\left.\mathrm{CDCl}_{3}\right) \delta: 0.72\left(1.5 \mathrm{H}, \mathrm{t}, \quad J=7.5 \mathrm{~Hz}, \mathrm{COOCH}\left(\mathrm{CH}_{3}\right) \mathrm{CH}_{2} \mathrm{CH}_{3}\right), 0.77(1.5 \mathrm{H}, \mathrm{t}, \quad J=7.5 \mathrm{~Hz}$, $\left.\mathrm{COOCH}\left(\mathrm{CH}_{3}\right) \mathrm{CH}_{2} \mathrm{CH}_{3}\right), 0.90\left(1.5 \mathrm{H}, \mathrm{d}, J=6.3 \mathrm{~Hz}, \mathrm{COOCH}\left(\mathrm{CH}_{3}\right) \mathrm{CH}_{2} \mathrm{CH}_{3}\right), 0.99(1.5 \mathrm{H}, \mathrm{d}, J=6.3 \mathrm{~Hz}$, COOCH $\left.\left(\mathrm{CH}_{3}\right) \mathrm{CH}_{2} \mathrm{CH}_{3}\right), 1.19-1.37$ (2H, m, COOCH$\left.\left(\mathrm{CH}_{3}\right) \mathrm{CH}_{2} \mathrm{CH}_{3}\right), 1.98$ (1.5H, s, $\left.\mathrm{OCOCH}_{3}\right), 1.98$ (1.5H, s, $\left.\mathrm{OCOCH}_{3}\right), 3.63\left(1.5 \mathrm{H}, \mathrm{s}, \mathrm{OCH}_{3}\right), 3.66\left(1.5 \mathrm{H}, \mathrm{s}, \mathrm{OCH}_{3}\right), 3.92-3.95\left(12 \mathrm{H}, \mathrm{s}, 4 \times \mathrm{OCH}_{3}\right), 4.63-4.82(3 \mathrm{H}, \mathrm{m}$, $\mathrm{ArCH}_{2} \mathrm{OAc}$ and $\left.\mathrm{COOCH}\left(\mathrm{CH}_{3}\right) \mathrm{CH}_{2} \mathrm{CH}_{3}\right), 5.74(0.5 \mathrm{H}, \mathrm{s}, \mathrm{OH}), 5.75(0.5 \mathrm{H}, \mathrm{s}, \mathrm{OH}), 6.59(0.5 \mathrm{H}, \mathrm{s}, 5-\mathrm{H})$, $6.61(0.5 \mathrm{H}, \mathrm{s}, 5-\mathrm{H}), 7.37$ (0.5H, s, 5'-H), $7.39\left(0.5 \mathrm{H}, \mathrm{s}, 5^{\prime}-\mathrm{H}\right) . \quad$ IR $(\mathrm{KBr}) \mathrm{cm}^{-1}: 3440(\mathrm{OH}), 1730(\mathrm{C}=\mathrm{O})$, 1695 (C=O), 1490, 1460, 1400, 1360, 1325, 1270, 1230, 1110, 1100, 1020, 1010. Anal. Calcd for $\mathrm{C}_{25} \mathrm{H}_{32} \mathrm{O}_{10}$ : C, 60.97; H, 6.55. Found: C, 61.07; H, 6.34.

\section{5-Acetoxymethyl-2,3-dimethoxyphenyl 2-iodo-4,5-dimethoxybenzoate}

A solution of $6^{7}$ (2.47 g, $\left.8.02 \mathrm{mmol}\right), 7^{8}$ (1.65 g, $\left.7.29 \mathrm{mmol}\right)$, EDC (1.40 g, $\left.7.29 \mathrm{mmol}\right)$, and DMAP (178 mg, $1.46 \mathrm{mmol})$ in $\mathrm{CH}_{2} \mathrm{Cl}_{2}(70 \mathrm{~mL})$ was stirred for $1 \mathrm{~h}$ at $\mathrm{rt}$, and then the mixture was poured into water and extracted with $\mathrm{CH}_{2} \mathrm{Cl}_{2}$. The organic layer was washed with brine and dried over magnesium sulfate. After evaporation, the resulting residue was purified by silica-gel column chromatography using hexaneEtOAc to give colorless needles (3.37 g, 90\%), mp 100-102 ${ }^{\circ} \mathrm{C}\left(\mathrm{Et}_{2} \mathrm{O}\right) .{ }^{1} \mathrm{H}_{-\mathrm{NMR}}\left(300 \mathrm{MHz}, \mathrm{CDCl}_{3}\right) \delta$ : $2.12\left(3 \mathrm{H}, \mathrm{s}, \mathrm{OCOCH}_{3}\right), 3.87\left(3 \mathrm{H}, \mathrm{s}, \mathrm{OCH}_{3}\right), 3.94\left(3 \mathrm{H}, \mathrm{s}, \mathrm{OCH}_{3}\right), 3.96\left(3 \mathrm{H}, \mathrm{s}, \mathrm{OCH}_{3}\right), 3.96\left(3 \mathrm{H}, \mathrm{s}, \mathrm{OCH}_{3}\right)$, $5.06\left(2 \mathrm{H}, \mathrm{s}, \mathrm{OCH}_{2} \mathrm{Ar}\right), 6.85(2 \mathrm{H}, \mathrm{s}$, phenoxy-4,6-H), $7.46(1 \mathrm{H}, \mathrm{s}$, benzoate-3-H), $7.70(1 \mathrm{H}, \mathrm{s}$, benzoate-6-H). ${ }^{13} \mathrm{C}-\mathrm{NMR}\left(75 \mathrm{MHz}, \mathrm{CDCl}_{3}\right) \delta: 21.1,56.1,56.2,56.4,60.9,65.8,85.8,110.4,114.3$, 115.2, 123.9, 124.5, 131.3, 140.9, 143.8, 148.6, 152.3, 153.5, 153.5, 163.3. IR $(\mathrm{KBr}) \mathrm{cm}^{-1}: 1750(\mathrm{C}=\mathrm{O})$, $1730(\mathrm{C}=\mathrm{O}), 1510,1270,1250,1210,1175$, 1095 . Anal. Calcd for $\mathrm{C}_{20} \mathrm{H}_{21} \mathrm{IO}_{8}$ : C, 46.53; H, 4.10. Found: C, 46.47; H, 3.98. 
A mixture of the ester (500 mg, $0.968 \mathrm{mmol}), \mathrm{PdCl}_{2}\left(\mathrm{Ph}_{3} \mathrm{P}\right)_{2}(169 \mathrm{mg}, 0.242 \mathrm{mmol}$ ), and sodium acetate (158 mg, $1.94 \mathrm{mmol}$ ) was dried under reduced pressure for $3 \mathrm{~h}$. This was dissolved in DMA (15 mL), and the mixture was stirred for $5 \mathrm{~h}$ at $120^{\circ} \mathrm{C}$. After filtration, the mixture was extracted with ethyl acetate, and then the organic layer was washed with brine, dried over magnesium sulfate, and evaporated. The resulting residue was purified by silica-gel column chromatography using chloroform to give colorless needles (5, $244 \mathrm{mg}, 65 \%)$, mp 191-193 ${ }^{\circ} \mathrm{C}\left(\mathrm{CH}_{2} \mathrm{Cl}_{2}\right.$-hexane). ${ }^{1} \mathrm{H}-\mathrm{NMR}\left(300 \mathrm{MHz}, \mathrm{CDCl}_{3}\right) \delta: 2.17(3 \mathrm{H}, \mathrm{s}$, $\left.\mathrm{OCOCH}_{3}\right), 3.98\left(3 \mathrm{H}, \mathrm{s}, \mathrm{OCH}_{3}\right), 4.02\left(3 \mathrm{H}, \mathrm{s}, \mathrm{OCH}_{3}\right), 4.028\left(3 \mathrm{H}, \mathrm{s}, \mathrm{OCH}_{3}\right), 4.031\left(3 \mathrm{H}, \mathrm{s}, \mathrm{OCH}_{3}\right), 5.49(2 \mathrm{H}$, s, $\left.\mathrm{OCH}_{2} \mathrm{Ar}\right), 7.06(1 \mathrm{H}, \mathrm{s}, 2-\mathrm{H}), 7.63(1 \mathrm{H}, \mathrm{s}, 10-\mathrm{H}), 7.84(1 \mathrm{H}, \mathrm{s}, 7-\mathrm{H}) .{ }^{13} \mathrm{C}-\mathrm{NMR}\left(75 \mathrm{MHz}, \mathrm{CDCl}_{3}\right) \delta$ : 21.2, 56.1, 56.22, 56.25, 61.5, 66.3, 107.0, 110.9, 112.9, 113.5, 114.2, 125.9, 129.7, 137.1, 145.9, 148.9, 151.9, 154.3, 160.2, 170.3. IR (KBr) $\mathrm{cm}^{-1}: 1740(\mathrm{C}=\mathrm{O}), 1720(\mathrm{C}=\mathrm{O}), 1610,1500,1320,1250,1230$, 1150, 1100, 1030. Anal. Calcd for $\mathrm{C}_{20} \mathrm{H}_{20} \mathrm{O}_{8}$ :C, 61.85; H, 5.19. Found: C, 61.64; H, 5.12.

\section{ACKNOWLEDGEMENTS}

We thank the SC-NMR Laboratory of Okayama University for performing the NMR experiments.

\section{REFERENCES}

1. a) T. Tanahashi, M. Kuroishi, A. Kuwahara, N. Nagakura, and N. Hamada, Chem. Pharm. Bull., 1997, 45, 1183. b) U. Höller, G. M. König, and A. D. Wright, Eur. J. Org. Chem., 1999, 2949. c) E. Hormazabal, G. Schmeda-Hirschmann, L. Astudillo, J. Rodríguez, and C. Theoduloz, Z. Naturforsch, 2005, 60c, 11. d) Y. Takenaka, Ph.D Thesis, Okayama University, 2003. d) A. H. Ali, R. Edrada-Ebel, I. D. Indriani, V. Wray, W. E. G. Müller, F. Totzke, U. Zirrgiebel, C. Schächtele, M. H. G. Kubbutat, W. H. Lin, P. Proksch, and R. Ebel, J. Nat. Prod., 2008, 71, 972.

2. H. Abe, K. Nishioka, S. Takeda, M. Arai, Y. Takeuchi, and T. Harayama, Tetrahedron Lett., 2005, 46, 3197.

3. H. Abe, T. Fukumoto, K. Nishioka, M. Arai, Y. Takeuchi, and T. Harayama, Heterocycles, 2006, 69, 217.

4. a) T. Harayama, Heterocycles, 2005, 65, 697, and references cited therein. b) H. Abe and T. Harayama, Heterocycles, 2008, 75, 1305.

5. a) G. Bringmann, J. Hinrichs, P. Henschel, K. Peters, and E.-M. Peters, Synlett, 2000, 1822. b) G. Bringmann, J. Hinrichs, P. Henschel, J. Kraus, K. Peters, and E.-M. Peters, Eur. J. Org. Chem., 2002, 1096. c) H. Abe, S. Takeda, Y. Takeuchi, and T. Harayama, Heterocycles, 2003, 61, 521. d) F. Büttner, S. Bergemann, D. Guenard, R. Gust, G. Seitz, and S. Thoret, Bioorg. Med. Chem., 2005, 13, 3497. e) W. R. Chang, Y. S. Lo, C. Y. Lee, and M. J. Wu, Adv. Synth. Catal., 2008, 350, 1248.

6. H. Abe, M. Arai, K. Nishioka, T. Kida, K. Shioe, Y. Takeuchi, and T. Harayama, Heterocycles, 2008, 
76, 291.

7. D. M. Piatak, G. Flynn, K. Yim, and J. Roosenberg, J. Org. Chem., 1977, 42, 1068.

8. P. Allevi, P. Ciuffreda, A. Longo, and M. Anastasia, Tetrahedron: Asymmetry, 1998, 9, 2915. 